\title{
Direct Measurement of Anaerobic Biodegradability of Nonylphenol Ethoxylates (NPEOs)
}

\author{
Mohd Hafiz Puteh, David C. Stuckey, and Mohd Hafiz Dzarfan Othman
}

\begin{abstract}
The biodegradation of nonylphenol ethoxylates (NPEOs) surfactant often produces more refractory, toxic and estrogenic intermediates. In this study, simple analytical method for NPEOs determination based on High Performance Liquid Chromatography (HPLC) was developed and complete biodegradation of NPEOs was evaluated by "serum bottle method" that uses direct measure of biogas production in anaerobic condition. The reversed phase $\mathrm{C18}$ column was successfully used for detection of long chain NPEO $_{\text {ave9 }}$ (having an average of 9 EO units) and short chain NPEO $_{\text {ave } 1.5}$ (having an average of $1.5 \mathrm{EO}$ units) and nonylphenol (NP) at short retention time. However, the coelution of oligomers in reversed phase chromatography is unavoidable, thus the distinction between NPEO oligomers is not possible. In biodegradation study study, the long chain NPEO $_{\text {ave9 }}$ was partially mineralized at approximately $37 \%$ of the theoretical $\mathrm{CH}_{4}$ production in anaerobic condition. Nevertheless, the refractory intermediates of NPEO $_{\text {ave1.5 }}$ and nonylphenol adversely affects the glucoseutilizing methanogens under anaerobic condition, thereby hampering $\mathrm{CH}_{4}$ formation. It can be concluded that the anaerobic biodegradation of nonylphenolic compounds proceeded at a very slow rate due to their complex physicochemical properties.
\end{abstract}

Index Terms-Nonylphenol ethoxylates, endocrine disrupting compounds, anaerobic, biochemical methane potential.

\section{INTRODUCTION}

Nonylphenol ethoxylates (NPEOs) is one of the most widely used industrial surfactants. The compound consists of a mixture of NPEO oligomers having different numbers of hydrophilic ethoxylate (EO) units with a diverse arrangement of isomeric, branched-chain hydrophobic nonyl groups (Fig. 1). Of these, NPEOs owing an average EO units of $9\left(\mathrm{NPEO}_{\mathrm{ave}}\right)$, are well known for its commercial application. The biodegradation route of NPEOs is complex. Microbial attack on the molecule is commonly occurs at the end of hydrophilic EO chain, causing chain excision to produce lower molecular weight NPEOs such as

Manuscript received September 5, 2014; revised December 18, 2014 This work was financially and technically supported by the Fundamental Research Grant Scheme (Project Number: R.J130000.7809.4F282), Research University Grant - Tier 1 (Project Number: Q.J130000.2522.09H03) Exploratory Research Grant Scheme (Project Number: R.J130000.7842.4L104) and Research Management Centre, Universiti Teknologi Malaysia.

Mohd Hafiz Puteh is with the Department of Environmental Engineering, Universiti Teknologi Malaysia, 81310 Skudai, Johor, Malaysia (e-mail: mhafizputeh@utm.my).

David C. Stuckey is with the Department of Chemical Engineering and Chemical Technology, Imperial College London, London SW7 2AZ, United Kingdom (e-mail: d.stuckey@ac.ic.uk).

Mohd Hafiz Dzarfan Othman is with the Advanced Membrane Technology Research Centre (AMTEC), Universiti Teknologi Malaysia, 81310 Skudai, Johor, Malaysia (e-mail: hafiz@petroleum.utm.my). nonylphenol (NP) and NPEO mono to triethoxylates (NPEO1-3) [1]-[4]. These intermediates are identified as Endocrine Disrupting Compounds (EDCs); that they may trigger normal hormonal function in humans and animals. In fact, they are more likely to persist in the environment and becoming more toxic than their parent compounds.

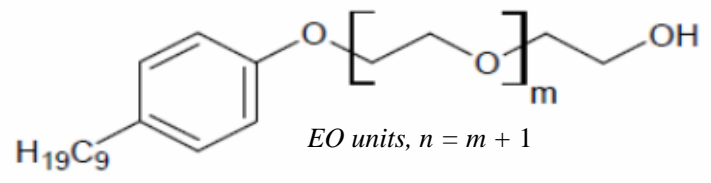

Fig. 1. Molecular structure of NPEOs.

Thus, the mineralisation or complete biodegradation of NPEOs to their simplest form is desired to protect the environment. It is well proven that the NPEOs can be degraded via oxidative (aerobic) and non-oxidative (anaerobic) pathways [3], [5]. There are good reasons for evaluating the anaerobic biodegradability of NPEOs since: (1) there is increasing demand for anaerobic treatment options in current sewage treatment plants due to biogas recovery; and (2) anaerobic treatment is effective for highstrength industrial wastewater, such as pulp and paper mill wastewater, in which significant amounts of NPEOs are commonly found [4], [6], [7]. The ultimate anaerobic biodegradation of a specific compound can be determined by measuring its disappearance or decrease, and the production of biogas using standard biodegradation assays [8]. The potential of a chemical to be degraded under anaerobic conditions, and its toxicity towards the anaerobic process, can be effectively monitored by the "serum bottle method" that uses direct measure of biogas production $\left(\mathrm{CH}_{4}\right.$ and $\left.\mathrm{CO}_{2}\right)$. The method, that provides relatively simple and inexpensive way of evaluating anaerobic biodegradation, was first described by Owen et al. [9], namely the anaerobic toxicity assay (ATA) and biochemical methane potential (BMP). BMP is a measure of substrate biodegradability determined by monitoring cumulative methane production from a sample which is anaerobically incubated in a chemically defined medium. The ATA measures the adverse effect or toxicity of a compound on the rate of the total gas production from an easily-utilized, methanogenic substrate.

Analytical detection and quantification of these compounds is difficult due to their complex physicochemical properties. High Performance Liquid Chromatography (HPLC) method is a simple and powerful tool in analyzing a broad range of NPEOs polarity. The presence of a phenol ring chromophore on NPEOs molecule enables their direct UV absorbance (at 277-280 nm) [10]. Thus, HPLC-UV method has been widely used under different elution modes (e.g. reversed and normal phase) for 
separation, detection and quantification of these complex compounds.

This study was aimed to develop a simple analytical method for NPEOs determination and to evaluate the anaerobic biodegradation of NPEOs using the serum bottle method.

\section{PROCEDURE}

\section{A. Materials}

The mixed-isomer technical grade nonylphenol (NP) and NPEOs surfactant mixtures of IGEPAL CO-630 and IGEPAL CO-210 contained branched-chain nonylphenol attached to ethylene oxide (EO) chains averaging 9 and 1.5 $\mathrm{EO}$ units, and collectively referred to as $\mathrm{NPEO}_{\mathrm{ave} 9}$ and $\mathrm{NPEO}_{\text {ave1.5, }}$, respectively, were obtained from Sigma Aldrich (Dorset, UK). The seed sludge was obtained from a sewage treatment plant (Mogden, West London, UK). The sludge was then inoculated into a $2 \mathrm{~L}$ Continuous Stirred Tank Reactor (CSTR) under anaerobic condition, supplemented with defined nutrient media as Owen et al. [9].

\section{B. Serum Bottle Methods}

Serum bottle studies will be conducted to evaluate anaerobic biodegradability of NPEOs based on BMP and ATA methods [9]. The study involves the use of $160 \mathrm{~mL}$ serum bottles, seeded with acclimatized biomass, feed with NPEOs stock solution, and supplied with defined media containing nutrients and vitamins for microbial cultures. The schematic diagram of the procedure is depicted in Fig. 2. The bottle is flushed thoroughly with a gas mixture of $70 \%$ $\mathrm{N} 2$ and $30 \% \mathrm{CO}_{2}$ under strict anaerobic conditions; before capping it with a leak proof Teflon seal and incubated at $37^{\circ} \mathrm{C}$. The volumetric gas production is measured by the syringe displacement method, and the biogas $\left(\mathrm{CH}_{4}\right.$ and $\left.\mathrm{CO}_{2}\right)$ composition is analyzed by gas chromatography (GC).

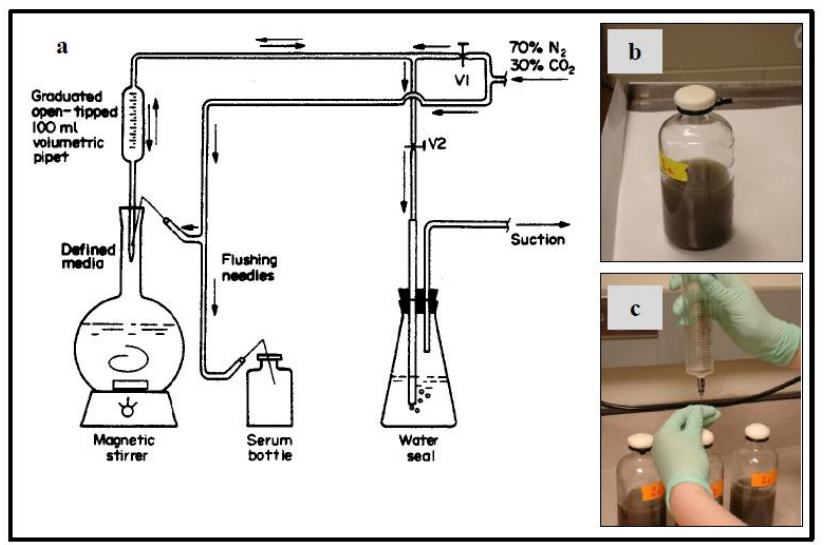

Fig. 2. (a) Schematic diagram of procedure for the anaerobic transfer of defined media into serum bottles; (b) Serum bottle; (c) Syringe displacement method for biogas volume measurement.

\section{Analytical Methods}

The analysis of nonylphenolic compounds was carried out using a LC-10Avp HPLC system (Shimadzu Co., Kyoto, Japan) consisting of two LC-10AS pumps, a SCL-10A system controller, a SIL-10AXL auto injector and a SPD10A UV-Vis absorption detector. The separation was performed under isocratic gradient elution using a $250 \times 4$ mm I.D., 5 $\mu \mathrm{m}$, Nucleosil C18 column (Macharey-Nagel, Duren, Germany). The biogas composition $\left(\mathrm{CH}_{4}\right.$ and $\left.\mathrm{CO}_{2}\right)$ was monitored using a Shimadzu Gas Chromatograph (GC) equipped with a TCD detector and fitted with a Porapak N column $(1500 \mathrm{~mm} \times 6.35 \mathrm{~mm})$. The carrier gas was helium, at a flow rate of $50 \mathrm{~mL} / \mathrm{min}$. The temperature of column, detector and injector were $28^{\circ} \mathrm{C}, 38^{\circ} \mathrm{C}$ and $128^{\circ} \mathrm{C}$, respectively. One $\mathrm{mL}$ of gas sample was manually injected into the GC injection port using $1 \mathrm{~mL}$ plastic syringes (Terumo).

\section{RESUlT AND DisCUSSION}

Fig. 3(a) and 3(b) display $\mathrm{C} 18$ reversed phase chromatograms commercial mixtures of $\mathrm{NPEO}_{\text {ave9 }}$, $\mathrm{NPEO}_{\text {ave } 1.5}$ and NP. Isocratic elution was performed with a mixture of $80 \%$ acetonitrile and $20 \%$ water; after trials, these were best results obtained using a Nucleosil C18 column. From the individual chromatograms, it can be seen that NPEOs and NP can be retained in the column and eluted in a short retention time. Nevertheless, the co-elution of $\mathrm{NPEO}_{\mathrm{ave} 9}, \mathrm{NPEO}_{\text {ave1.5 }}$ peaks is observed (Fig. 3(b)).
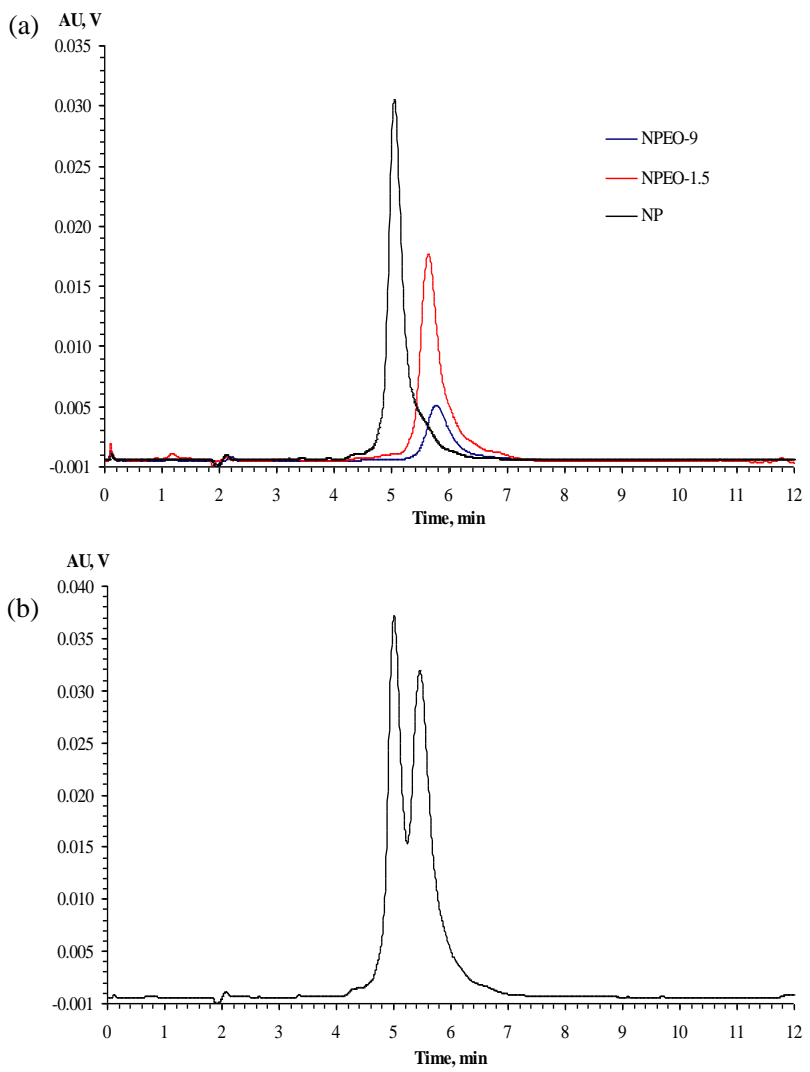

Fig. 3. (a) Chromatograms of individual NPEO-9, NPEO-1.5 and NP commercial standard solutions; (b) Separation of a mixture of commercial NPEO-9, NPEO-1.5 and NP.

It is important to note that the manufactured commercial ethoxylated nonylphenols consist of a broadly distributed mixture of oligomers of ethoxylated components. These oligomers refer to the number of polar (hydrophilic) ethoxylate chains (polyoxyethylene glycol) per molecule, usually ranging from 2 to about 20 units [10]. Meanwhile, the non-polar or hydrophobic region consists of a linear or branched nonylphenol. Therefore, the non-polar reversedphase C18 column is able to separate the NPEOs by their 
interaction with the hydrophobic chain, thus eluting the ethoxymers as a single peak [11]. This coelution has often been claimed to be an advantage, since it would increase the sensitivity of detection.

The BMP and ATA assay developed by Owen et al. [9] was used for the evaluation of NPEO anaerobic degradation. Fig. 4 provides a representative set of the cumulative methane production data obtained with the BMP test for the blank (biomass) and the serum bottle containing $20 \mathrm{mg} / \mathrm{L}$ $\mathrm{NPEO}_{\mathrm{ave} 9}$ with biomass. No source of organic matter or electron acceptor is added as the $\mathrm{NPEO}_{\text {ave9 }}$ acts as a sole carbon source. As can be seen from the figure, only a slight difference in gas production between the sample (serum bottle containing $\mathrm{NPEO}_{\mathrm{ave}}$ with biomass) and the blank (serum bottle containing only biomass) was observed. This may be due to small concentration of $\mathrm{NPEO}_{\mathrm{ave}}$, which was too low to generate substantially amounts of $\mathrm{CH} 4$ production. The $\mathrm{CH}_{4}$ production for the $\mathrm{NPEO}_{\text {ave9 }}$ was slightly higher compared to that of the blank, indicating the $\mathrm{CH}_{4}$ production from the degradation of $\mathrm{NPEO}_{\text {ave9 }}$. Theoretical gas production can be calculated by the Buswell equation, based on the stoichiometry of the reaction [12]. From the experimental result, calculating from the difference in cumulative gas production to the blank, the $\mathrm{NPEO}_{\text {ave9 }}$ was found to be partially mineralized (approximately $37 \%$ of theoretical methane production) under the given conditions. This was due to compounds' inhibitory nature. In addition, Ejlertsson et al. [3] suggested that the phenol ring structure remained intact during the period of incubation, while the EO units liberated as ethylene glycol during the degradation of long chain NPEO could have been further degraded to $\mathrm{CH}_{4}$ and $\mathrm{CO}_{2}$.

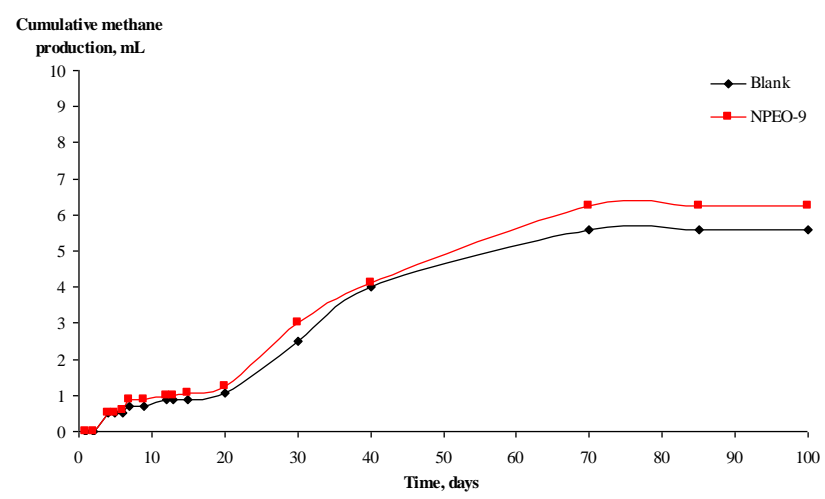

Fig. 4. Methane production from anaerobic $\mathrm{NPEO}_{\text {ave }}$ degradation.

Inhibition indicates a detrimental effect that a test substance causes on the activity of a microbial population. In the subsequent study, the inhibition of $\mathrm{NPEO}_{\mathrm{ave} 9}$ and its intermediates, $\mathrm{NPEO}_{\text {ave } 1.5}$ and $\mathrm{NP}$ were assessed in terms of $\mathrm{CH}_{4}$ production compared to that of a blank; the results are shown in Fig. 5. This ATA test conditions were similar to that of prior BMP assays, except that all bottles were spiked with $100 \mathrm{mg} / \mathrm{L}$ of test substance $\left(\mathrm{NPEO}_{\mathrm{ave}} 9, \mathrm{NPEO}_{\text {ave } 1.5}\right.$ and $\mathrm{NP}$ ) and $1.5 \mathrm{~g} / \mathrm{L}$ of easily degradable glucose. It is clearly seen from the figure that almost no $\mathrm{CH}_{4}$ was detected for $\mathrm{NPEO}_{\text {ave1.5 }}$ and NP. Meanwhile, the glucose was successfully degraded in the presence of $\mathrm{NPEO}_{\mathrm{ave}}$. This indicates that the $\mathrm{NPEO}_{\text {ave } 1.5}$ and NP adversely affected the glucose-utilizing methanogens, thereby hampering $\mathrm{CH}_{4}$ formation. It is known that high initial concentrations of NP have a toxic effect on the microorganisms [11]. Moreover, Lu et al. [13] observed the inhibition of anaerobic biodegradation of NPEO only when NP was added at relatively high concentrations. However, it is difficult to access the degradation of low concentrations of NPEO and its intermediates by means of gas production. Therefore, quantification of compounds in terms of concentration removal is necessary.

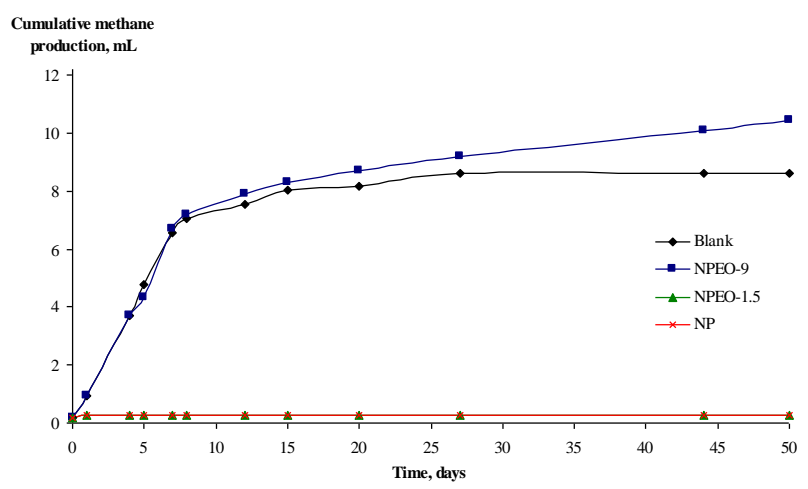

Fig. 5. Inhibition of methanogens by nonylphenolic compounds.

\section{CONCLUSION}

The reversed phase $\mathrm{C} 18$ column was successfully used for detection of NPEOs and NP at short retention time. However, the coelution of oligomers in reversed phase chromatography is unavoidable, thus the distinction between NPEO oligomers is not possible. The serum bottle studies indicate that the given concentrations of $\mathrm{NPEO}_{\text {ave1.5 }}$ and $\mathrm{NP}$ adversely affected the glucose-utilizing methanogens under anaerobic condition, thereby hampering $\mathrm{CH}_{4}$ formation. Although $\mathrm{NPEO}_{\text {ave9 }}$ did not inhibit the $\mathrm{CH}_{4}$ formation, the anaerobic reaction however proceeded at a very slow rate.

\section{ACKNOWLEDGMENT}

The authors gratefully acknowledge financial support from the Fundamental Research Grant Scheme (Project Number: R.J130000.7809.4F282), Research University Grant - Tier 1 (Project Number: Q.J130000.2522.09H03) and Exploratory Research Grant Scheme (Project Number: R.J130000.7842.4L104); and technical support from Research Management Centre, Universiti Teknologi Malaysia.

\section{REFERENCES}

[1] W. Giger, P. H. Brunner, and C. Schaffner, "4-Nonylphenol in sewage sludge: accumulation of toxic metabolites from non-ionic surfactants," Science, vol. 225, pp. 623-625, 1984.

[2] C. A. Staples, J. B. Williams, R. L. Blessing, and P. T. Varineau, "Measuring the biodegradability of nonylphenol ether carboxylates, octylphenol ether carboxylates, and nonylphenol," Chemosphere, vol. 38, pp. 2029-2039, 1998.

[3] J. Ejlertsson, M. L. Nilsson, H. Kylin, A. Bergman, L. Karlson, and M. Oquist, "Anaerobic degradation of nonylphenol mono-and diethoxylates in digester sludge, landfill municipal solid waste and landfill sludge," Environmental Science and Technology, vol. 33, pp. 301-306, 1999.

[4] J. Zhang, M. Yang, Y. Zhang, and M. X. Chen, "Biotransformation of nonylphenol ethoxylates during sewage treatment under anaerobic and aerobic conditions," Journal of Environmental Sciences, vol. 20, pp. $135-141,2008$. 
[5] S. Hayashi, S. Saito, J. H. Kim, O. Nishimura, and R. Sudo, "Aerobic biodegradation behavior of nonylphenol polyethoxylates and their metabolites in the presence of organic matter," Environmental Science and Technology, vol. 39, pp. 5626-5633, 2005.

[6] K. Strevett, I. Davidova, and J. M. Suflita, "A comprehensive review of the screening methodology for anaerobic biodegradability of surfactants," Reviews in Environmental Science and Biotechnology, vol. 1, pp. 143-167, 2002.

[7] M. Hawrelak, E. Bennett, and M. Metcalfe, "The environmental fate of the primary degradation products of alkylphenol ethoxylate surfactants in recycled paper sludge," Chemosphere, vol. 39, pp. 745752, 1999.

[8] C. A. Staples, C. G. Naylor, and J. B. Williams, "Ultimate biodegradability of C8- and C9-alkylphenol ethoxylate degradation intermediates," Environmental Toxicology and Chemistry, vol. 20, pp. 2450-2455, 2001.

[9] W. F. Owen, D. C. Stuckey, J. B. Healy Jr., L. Y. Young, and P. L. McCarty, "Bioassay for monitoring biochemical methane potential and anaerobic toxicity," Water Research, vol. 13, pp. 485-492, 1979.

[10] A. T. Kiewiet and E. de Voogt, "Chromatographic tools for analyzing and tracking non-ionic surfactants in the aquatic environment," Journal of Chromatography A, vol. 733, pp. 185-192, 1996.

[11] B. Thiele, K. Gunther, and M. J. Schwuger, "Alkylphenol ethoxylates: trace analysis and environmental behavior," Chemical Reviews, vol. 97, pp. 3247-3272, 1997.

[12] D. R. Shelton and J. M. Tiedje, "General method for determining anaerobic biodegradation potential," Applied and Environmental Microbiology, vol. 47, pp. 850-857, 1984.

[13] J. Lu, Q. Jin, Y. L. He, and J. Wu, "Biodegradation of nonylphenol polyethoxylates under $\mathrm{Fe}(\mathrm{III})$-reducing conditions," Chemosphere, vol. 69, pp. 1047-1054, 2007.

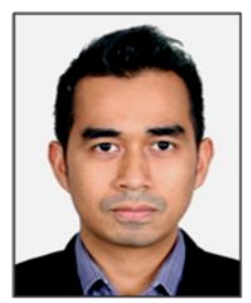

Mohd Hafiz Puteh graduated with a B.Eng. degree in chemical engineering and a master degree of engineering in enviromental from Universiti Teknologi Malaysia (UTM) in 2004 and 2006 respectively. He started his academic career when he was appointed as a tutor in 2006 in the Department of Environmental Engineering, Faculty of Civil Engineering. Shortly after that, he was granted with a scholarship from Ministry of Higher Education to further his PhD study in chemical engineering at Imperial College London, focusing on biodegradation of nonylphenol ethoxylates (NPEOs) in a membrane aerated biofilm reactor (MABR). He is now actively involved in 'Research and Innovation' and 'Teaching and Learning' fields.

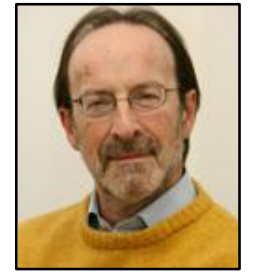

David Stuckey has established an international reputation in the area of biological wastewater treatment, particularly in the field of anaerobic digestion. When he was a graduate student, he developed the standard bioassays in this field, the Biochemical Methane Potential (ATA) and Anaerobic Toxicity Assay (ATA) which are now used throughout the World. He went on to develop two novel reactor types; the Anaerobic Baffled Reactor (ABR), and the Submerged Anaerobic Membrane Bioreactor (SAMBR), the former with over 700 units throughout the World. $\mathrm{He}$ obtained his B.Eng degree in chemical engineering, and M.Eng Sci. degree in biochemical engineering from the University of Melbourne, Australia After working in the State Government for a few years, he went to California to do his PhD study at Stanford with Prof Perry McCarty, recipient of the Stockholm Water Prize. After Postdocs at NTH in Trondheim, and EAWAG in Zurich, he joined Chemical Engineering at Imperial College London where he established substantial activities in both biochemical and environmental engineering, and is currently a professor in biochemical engineering. He has published over 130 technical papers, edited one book, and written a number of book chapters. He sits on the Editorial Board of 5 Journals. Recently (2008) he was awarded the prestigious Royal Society Brian Mercer Award for Innovation to pilot the SAMBR for treating sewage, while recovering energy and reducing solids production.

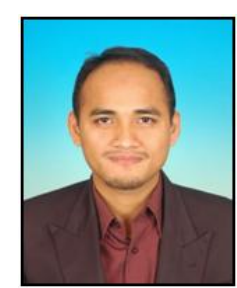

Mohd Hafiz Dzarfan graduated with the first class of bachelor degree of science in chemical engineering from Universiti Teknologi Malaysia (UTM) and began his academic career as a tutor at the Department of Gas Engineering in UTM in 2004. In 2006, he successfully obtained his master of engineering degree with the publication of one article paper in international journal and two articles in national journal, before was appointed as a lecturer in UTM in the same year. In the end of 2007, he pursued his PhD study in Imperial College London and had been working on the project of "High Performance Micro-Tubular Solid Oxide Fuel Cell". In this study, he was successfully developed a novel, high quality hollow fibre support for an energy conversion device, so-called micro-tubular solid oxide fuel cells (SOFCs), using a more economical and reliable technique, i.e., a phase inversion-based co-extrusion process. During the period of his $\mathrm{PhD}$ study, he managed to publish 11 articles papers in high-reputation journals and 1 article in book chapter. After coming back from PhD study, he continues his interest in membrane research by starting a new research area in Malaysia, which is more focus in ceramic membrane for high temperature purpose. By doing this, he wishes he could explore more challenging and interesting research areas that more suits to the applications in Malaysia. 